\title{
Loss of Function of the Gene Encoding the Histone Methyltransferase KMT2D Leads to Deregulation of Mitochondrial Respiration
}

\author{
Consiglia Pacelli ${ }^{1}{ }^{\oplus}$, Iolanda Adipietro ${ }^{2}$, Natascia Malerba ${ }^{2}$, Gabriella Maria Squeo ${ }^{2}$, \\ Claudia Piccoli ${ }^{1}$, Angela Amoresano ${ }^{3,4}$, Gabriella Pinto ${ }^{3} \mathbb{D}$, Pietro Pucci ${ }^{3,5}$, Ji-Eun Lee ${ }^{6} \mathbb{D}$, \\ Kai Ge ${ }^{6} \mathbb{D}$, Nazzareno Capitanio ${ }^{1, *}$ and Giuseppe Merla ${ }^{2, * \mathbb{D}}$ \\ 1 Department of Clinical and Experimental Medicine, University of Foggia, 71121 Foggia, Italy; \\ consiglia.pacelli@unifg.it (C.P.); claudia.piccoli@unifg.it (C.P.) \\ 2 Division of Medical Genetics, Fondazione IRCCS Casa Sollievo della Sofferenza, \\ 71013 San Giovanni Rotondo (FG), Italy; iole.adipietro@gmail.com (I.A.); \\ n.malerba@operapadrepio.it (N.M.); g.squeo@operapadrepio.it (G.M.S.) \\ 3 Department of Chemical Sciences, University of Naples "Federico II", 80126 Napoli, Italy; \\ angela.amoresano@unina.it (A.A.); gabriella.pinto@unina.it (G.P.); pucci@unina.it (P.P.) \\ 4 INBB-Biostructures and Biosystems National Institute, 00136 Roma, Italy \\ 5 Department of Chemical Sciences, CEINGE Advanced Biotechnology, Federico II University, \\ 80145 Napoli, Italy \\ 6 National Institute of Diabetes and Digestive and Kidney Diseases, National Institutes of Health, \\ Bethesda, MD 20892, USA; leejieun@niddk.nih.gov (J.E.L.); kaig@niddk.nih.gov (K.G.) \\ * Correspondence: nazzareno.capitanio@unifg.it (N.C.); g.merla@operapadrepio.it (G.M.)
}

Received: 18 May 2020; Accepted: 9 July 2020; Published: 13 July 2020

\begin{abstract}
KMT2D encodes a methyltransferase responsible for histone 3 lysine 4 (H3K4) mono-/dimethylation, an epigenetic mark correlated with active transcription. Here, we tested the hypothesis that KMT2D pathogenic loss-of-function variants, which causes the Kabuki syndrome type 1, could affect the mitochondrial metabolic profile. By using Seahorse technology, we showed a significant reduction of the mitochondrial oxygen consumption rate as well as a reduction of the glycolytic flux in both Kmt2d knockout MEFs and skin fibroblasts of Kabuki patients harboring heterozygous KMT2D pathogenic variants. Mass-spectrometry analysis of intermediate metabolites confirmed alterations in the glycolytic and TCA cycle pathways. The observed metabolic phenotype was accompanied by a significant increase in the production of reactive oxygen species. Measurements of the specific activities of the mitochondrial respiratory chain complexes revealed significant inhibition of CI (NADH dehydrogenase) and CIV (cytochrome c oxidase); this result was further supported by a decrease in the protein content of both complexes. Finally, we unveiled an impaired oxidation of glucose and larger reliance on long-chain fatty acids oxidation. Altogether, our findings clearly indicate a rewiring of the mitochondrial metabolic phenotype in the KMT2D-null or loss-of-function context that might contribute to the development of Kabuki disease, and represents metabolic reprogramming as a potential new therapeutic approach.
\end{abstract}

Keywords: Kabuki syndrome; KMT2D; mitochondria

\section{Introduction}

Growing evidence highlights the tight interaction between metabolism and epigenetics [1]. Covalent modification of DNA and histones require, indeed, the availability of amino acid derivatives, cofactors, and central metabolites to feed the plethora of epigenetic-modifying enzymes. Other ways 
to induce epigenetic modulations, such as chromatin remodeling, also impact the metabolic phenotype. However, the mechanisms that confer selectivity of chromatin remodeling in regulating the metabolic phenotype remain to be fully understood.

Histone-lysine N-methyltransferase 2D (KMT2D) belongs to the SET1 family of histone H3K4 methyltransferases and is part of the ASCOM multi-protein complex that catalyzes mono-, di-, and tri-methylation of H3K4 using S-adenosyl methionine as a co-substrate [2]. KMT2D is a positive key regulator of gene expression in the context of cellular differentiation in different tissues. Although KMT2D activity can be replaced by other histone methyltransferases, its genetic knockout reduced global H3K4me1 levels, and the majority of KMT2D binding sites are localized in putative enhancer elements [3-5].

Pathogenic loss-of-function (lof) variants in the KMT2D gene with an autosomal dominant pattern of inheritance have been linked to the great majority of cases of Kabuki syndrome, which is one of the most well-characterized pediatric chromatinopathies [6] and is hallmarked by multiple congenital anomalies and intellectual disabilities [7,8]. A lower percentage of cases of Kabuki syndrome are related to pathogenic variants in the KDM6A gene, which encodes another important component of the ASCOM complex that is responsible for H3K27 demethylation of repressive polycomb-derived methylation marks [9]. Studies in mouse models have demonstrated that $K m t 2 d$ is essential for adipogenesis, myogenesis, macrophage activation, and lymphomagenesis, demonstrating additional roles for KMT2D as a mono- and di-methyltransferase at enhancers $[5,10,11]$. Collectively, these findings indicate that KMT2D regulates key gene expression programs via H3K4 mono-, di-, and tri-methylation, and its role depends on the cellular context and developmental stage. Intriguingly, heterozygous $\mathrm{Kmt} 2 \mathrm{~d}^{+/-}$mice exhibit metabolic alterations consisting of enhanced glucose tolerance, insulin sensitivity, and increased serum bile acid [12] as well as resistance to over-nutrition-induced hepatic steatosis [13]. These lines of evidences suggest a regulatory function of KMT2D-mediated epigenetic modification on metabolism. Since mitochondria have emerged as an important source of metabolites utilized as co-substrates by both epigenetic writers and erasers [14], we investigated this issue by focusing on the role of KMT2D in mitochondrial aerobic metabolism. For this purpose, we used Kmt2d knockout MEFs and fibroblasts from Kabuki syndrome-affected patients harboring KMT2D heterozygous pathogenic variants. By combining biochemical and metabolomic approaches in mouse and human cell models, we demonstrated a rewiring of the mitochondrial metabolic phenotype due to KMT2D alteration that might contribute to the onset of Kabuki syndrome.

\section{Materials and Methods}

\subsection{Cell Samples}

Primary dermal fibroblasts were obtained from skin biopsies of Kabuki patients and healthy individuals after signing the appropriate informed consent, provided by Genomic and Genetic Disorders Biobank, member of the Telethon Network of Genetic Biobanks [15]. The generation and use of skin fibroblasts were reviewed and approved by Ethical Committee at Fondazione IRCCS Casa Sollievo della Sofferenza (14/11/2018, 156/CE). Cells were grown in minimum essential medium supplemented with $1 \%$ glutamine, $10 \%$ FBS, and $1 \%$ PenStrep. Healthy control skin fibroblast was transiently transfected with $2 \mu \mathrm{g}$ of MLL2 CRISPR/Cas9 KO Plasmids (Santa Cruz Biotechnology, Dallas, TX, USA). After 48 h, GFP-positive transfected cells were enriched by FACS analysis and isolated as a single cell in a 96 multi-well plate. After approximately 20 days, single-well adherent cells were detached by trypsinization, and expanded as clonal cells. Each clone was analyzed for KMT2D variants by Sanger sequencing. The presence of off-targets predicted by Cas-OFFinder (http://www.rgenome.net/cas-offinder/) using default parameters [16] was ruled out by PCR and Sanger sequencing (Table S1).

Primary mouse embryonic fibroblasts (MEFs) isolated from E13.5 Kmt2df/f embryos [5] were immortalized by following the $3 \mathrm{~T} 3$ protocol. Immortalized $K m+2 d^{\mathrm{f} / \mathrm{f}} \mathrm{MEFs}$ were infected with 
retroviruses expressing Cre to generate Kmt2d-KO MEFs. Cells were cultured in DMEM supplemented with $10 \%$ FBS and $1 \%$ PenStrep.

\subsection{Laser Scanning Confocal Microscopy Live Imaging}

Cells cultured at a low density on fibronectin-coated 35-mm glass-bottom dishes (Ibidi) were incubated for $20 \mathrm{~min}$ at $37^{\circ} \mathrm{C}$ with $0.2 \mu \mathrm{M}$ tetramethylrhodamine ethyl ester (TMRE, Invitrogen, Molecular probesTM, Carlsbad, CA, USA) to detect the mitochondrial membrane potential, or with $10 \mu \mathrm{M}$ 2,7-dichlorofluorescin diacetate (DCF-DA, Invitrogen, Molecular probesTM, Carlsbad, CA, USA ) to detect cellular peroxide, or with $5 \mu \mathrm{M}$ MitoSOX ${ }^{\mathrm{TM}}$ (Invitrogen, Molecular probesTM, Carlsbad, CA, USA) to detect mitochondrial superoxide anion. Stained cells were washed with PBS and examined by a Leica TCS SP8 confocal laser scanning microscopy system (images collected using a 60X objective [1.4 NA]). Acquisition, storage, and analysis of data were performed with LasX software from Leica or ImageJ1.48 (Wayne Rasband, NIH, USA, http://imagej.nih.gov/ij).

\subsection{Immunoblotting}

Immunoblots were performed by standard protocol; PVDF membranes were probed with MitoProfile Total OXPHOS Human WB Antibody cocktail (1:500; Abcam Cambridge, UK) and $\beta$-ACTIN (1:5000; SIGMA Aldrich, St. Louis, MO, USA). The secondary antibody was horseradish peroxidase-conjugated (1:2500; Cell Signaling Technology). The signals were developed by an enhanced chemiluminescence kit (ClarityTM Western ECL Substrate, Bio-Rad), acquired by a ChemiDoc Imaging System XRS + (BioRad), and then analyzed for densitometry with the ImageJ Lab 4.1 software.

\subsection{RNA Isolation and Quantitative RT-PCR (RT-qPCR)}

Total RNA was extracted using the RNeasy Mini Kit (Qiagen, Germany). The QuantiTect Reverse Transcription Kit (Qiagen) was used to carry out cDNA synthesis. cDNA was amplified using Power SYBR Green PCR Master Mix (Applied biosystem) on an ABI PRISM 7009HT System. The relative amounts of target genes were normalized to GAPDH or RN18S and the $2^{-\Delta \Delta \mathrm{Ct}}$ method was used for calculations. Validated primers for RT-qPCR are provided upon request.

\subsection{Metabolites Extraction}

A liquid-liquid extraction was carried out for the recovery of metabolites and protein precipitation from whole lysates of wild-type $(+/+)$, heterozygous mutant $(+/-)$, and homozygous mutant $(-/-)$ human cells. In detail, a solution of cold acetonitrile/methanol (50/50) containing $0.1 \%$ acetic acid was used to dilute the cellular lysates by 5 -fold. The suspension was sonicated for $10 \mathrm{~min}$, cooled at $-20^{\circ} \mathrm{C}$, and centrifuged at $12,000 \mathrm{rpm}$ for $10 \mathrm{~min}$ at $4{ }^{\circ} \mathrm{C}$. The supernatant was filtered by $0.22-\mu \mathrm{m}$ centrifugal filters (PVDF, Millipore) and analyzed by a LC-MS/MS mass spectrometer. Each sample was analyzed in duplicate. Three biological replicates were analyzed for heterozygous and homozygous mutants, and two biological replicates were analyzed for wild-type ones.

\subsection{LC-MS/MS Analysis}

Metabolite extracts were separated by liquid chromatography using an LC Eksigent combined to a 4000 QTRAP@mass spectrometer (AB Sciex). The auto-sampler was cooled at $4{ }^{\circ} \mathrm{C}$, the injection volume was $5 \mu \mathrm{L}$, and the flow rate was set to $30 \mu \mathrm{L} / \mathrm{min}$. The chromatographic separation was obtained on a column Halo C18 $2.7 \mathrm{um} 90 \mathrm{~A} 1 \times 50 \mathrm{~mm}$ at a temperature of $38^{\circ} \mathrm{C}$. Eluent $\mathrm{A}$ was $\mathrm{H}_{2} \mathrm{O}$ and $0.1 \%$ acetic acid, whereas eluent $\mathrm{B}$ was a solution of $50 \% \mathrm{ACN}, 50 \%$ isopropanol, and $0.1 \%$ acid acetic. Gradient conditions followed an increase of organic eluent from $0 \%$ to $90 \%$ in a 6 -min run. The mass spectrometry analysis was performed in multiple reaction monitoring (MRM) mode, with an ionization source ESI. Metabolites were tuned for negative ionization polarity by using optimized 
values of precursor (Q1) and daughter ions (Q2) as well as collision energy (CE) and declustering potential (DP) as reported in the literature [17].

\subsection{Measurements of Metabolic Fluxes}

Cellular respiration (oxygen consumption rate (OCR) and extracellular acidification rate (ECAR)) were assessed using an XF96 Extracellular Flux Analyzer (Seahorse Bioscience, Billerica, MA, USA). First, $15 \times 10^{3} /$ well MEFs and $10 \times 10^{3} /$ well fibroblasts were incubated for $45 \mathrm{~min}$ in $180 \mu \mathrm{L}$ of bicarbonate-free DMEM supplemented with $10 \mathrm{mM}$ glucose $2 \mathrm{mM}$ l-glutamine and $1 \mathrm{mM}$ sodium pyruvate pre-warmed at $37^{\circ} \mathrm{C}$. The XF Cell Mito Stress Test (Seahorse Bioscience) was used to measure the key parameters of mitochondrial respiration when using specific mitochondrial inhibitors and uncouplers. Oligomycin $(1 \mu \mathrm{M})$, carbonilcyanide $p$-triflouromethoxyphenylhydrazone (FCCP) $(0.5 \mu \mathrm{M})$, and a mixture of rotenone/antimycin A (both $1 \mu \mathrm{M}$ ) were injected sequentially according to the manufacturer's instructions. Before drug addition, basal OCR was measured. Oligomycin was injected to inhibit ATP synthase (complex V), and OCR was recorded. To determine the maximal respiration, the uncoupler FCCP was injected. Finally, a mixture of rotenone/antimycin A was injected to inhibit the flux of electrons through complexes I and III and to enable the calculation of the spare respiratory capacity. For ECAR analysis, glycolytic flux (basal glycolysis, glycolytic capacity, and glycolytic reserve) was analyzed by the sequential addition of $10 \mathrm{mM}$ glucose, $1 \mu \mathrm{M}$ oligomycin, and $100 \mathrm{mM}$ 2-deoxyglucose. The Seahorse XF Mito Fuel Flex Test Kit was used for measuring the basal state mitochondrial fuel oxidation in live cells. This assay kit utilizes a set of inhibitors to reveal the cells' ability to switch oxidative pathways in meeting basal energetic demands and provides information regarding the relative contributions of glucose, glutamine, and long-chain fatty acid oxidation to basal respiration. The assay uses specific inhibitors in different combinations to measure the dependency, capacity, and flexibility of cells to oxidize 3 major mitochondrial fuels: Glucose (pyruvate), glutamine (glutamate), and long-chain fatty acids in the basal energetic state. The inhibitors used were: BPTES $(3 \mu \mathrm{M})$, an inhibitor of glutaminase; etomoxir $(4 \mu \mathrm{M})$, an inhibitor of long-chain fatty acid $\beta$-oxidation (FAO); and UK5099 $(2 \mu \mathrm{M})$, an inhibitor of the mitochondrial pyruvate carrier. All measurements were normalized to the total protein concentration using the Pierce BCA Protein Assay Kit (Thermo Fisher Scientific).

\subsection{Measurement of the Steady-State Content and Rates of Production of ATP}

Cellular steady-state ATP content was determined using the PerkinElmer ATPlite kit (PerkinElmer) according to the manufacturer's instructions on MEFs collected by trypsinization, pelleted at $500 \times g$, and resuspended in phosphate-saline buffer. To distinguish the relative contributions to the ATP production of glycolysis, cultured cells were treated with $1.0 \mu \mathrm{M}$ oligomycin for 10-30 min and the residual ATP level attributed to glycolysis; the difference between the ATP content measured in the absence and in the presence of oligomycin was attributed to the mitochondrial ATP-synthase. Measurements were performed with a TD 20-20 Luminometer (Turner Designs) and normalized to the protein content.

The cellular ATP production rates were quantified using the Agilent Seahorse XF ATP real-time assay kit using label-free technology in real time as specified by the manufacturer (see also [18]. Briefly, according to the equation: glucose $+2 \mathrm{ADP}+2 \mathrm{Pi} \rightarrow 2$ lactate $+2 \mathrm{ATP}+2 \mathrm{H}_{2} \mathrm{O}+2 \mathrm{H}^{+}$, the glycolytic ATP production rate (glycoATP PR) was estimated from the glycolytic proton efflux rate (glycoATP PER) following determination of the buffering power (BP). BP was calculated separately by measuring $\mathrm{pH}$ changes elicited by consecutive additions of titrated $\mathrm{HCl}$ in $5 \mathrm{mM}$ Hepes-supplemented DMEM, in the presence of the same number of cells assayed and was $1.79\left(\mathrm{mmol} \mathrm{H}^{+} / \mathrm{L} / \mathrm{pH}\right)$. The mitochondrial ATP production rate (mitoATP PR) was equal to OCR $\mathrm{ATP}_{\mathrm{A}} \times 2 \times \mathrm{P} / \mathrm{O}(\mathrm{mol} \mathrm{ATP} / \mathrm{mol} \mathrm{O})$, where OCR $\mathrm{R}_{\text {ATP }}$ was the difference between the basal OCR and the OCR in the presence of oligomycin; a theoretical $\mathrm{P} / \mathrm{O}$ ratio of 2.25 was assumed based on the combined oxidation of glucose, fatty acid, and glutamine. 


\subsection{Mitochondrial Respiratory Complex Enzymatic Activity}

Measurement of the specific activity of mitochondrial NADH:ubiquinone oxidoreductase (C I), cytochrome c oxidase (C IV), and citrate synthase (CS) was carried out spectrophotometrically on frozen-thawed and ultrasound-treated cells as previously described $[19,20]$.

\subsection{Statistical Analysis}

Data are shown as mean \pm SEM. Data were compared by an unpaired student's $\mathrm{t}$-test. Differences were considered statistically significant when the $p$-value was less than 0.05. All analyses were performed using Graph Pad Prism (Graph Pad software, v 6.01, San Diego, CA, USA).

\section{Results}

\subsection{Loss of Kmt2d Causes Deregulation of the Respiratory and Glycolytic Metabolic Fluxes}

As a first line of investigation, we sought to verify if the KMT2D loss of function impacted the bioenergetic cell metabolism. To this aim, we used the Seahorse methodology [21] to assess the two major metabolic fluxes in intact cells: i) Mitochondrial oxidative phosphorylation (OxPhos) activity, measured as the oxygen consumption rate (OCR); and ii) glycolysis, measured as the extracellular acidification rate (ECAR) largely contributed by lactate release. Figure 1 shows the results attained from comparing the OCR and ECAR activities between wild-type (WT) MEF and Kmt2d - knockout (KO) MEF. The values were corrected for residual activity in the presence of the mitochondrial respiratory chain inhibitors rotenone plus antymicin A for OCR (Figure 1A) and in the presence of the glycolysis inhibitor 2-deoxyglucose for ECAR (Figure 1C). The OCR under basal conditions was significantly reduced in $K m t 2 d-K O$ MEF ( $\approx 70 \%$ of WT-MEF). Following addition of the $\mathrm{H}^{+}$-ATP synthase inhibitor oligomycin, the OCR was depressed to a similar extent in both WT-MEF and Kmt2d-KO MEF, indicating no variation in the membrane proton leak (Figure 1B). Addition of the protonophore uncoupler FCCP stimulated the OCR to its maximal activity in both wt-MEF and Kmt2d-KO MEF but to a significantly lower extent in $K m t 2 d-K O$ MEF ( $\approx 55 \%$ of WT-MEF) (Figure 1B). Consequently, in Kmt2d-KO MEF, both the OCR linked to ATP synthesis (i.e., the difference between basal OCR and OCR plus-oligomycin), and the spare respiratory capacity (i.e., the difference between OCR plus-FCCP and basal OCR) were significantly reduced ( $\approx 85 \%$ and $\approx 39 \%$ of wt-MEF, respectively). As compared with WT-MEF, ECAR was slightly reduced in Kmt2d-KO MEF under the basal condition and more significantly reduced under conditions maximally stimulating glycolysis, achieved by oligomycin treatment (i.e., glycolytic capacity) (Figure 1D). As a result, the ECAR spare capacity (i.e., glycolytic reserve) was lower in Kmt2d-KO MEF. Altogether, these results suggest a reduced mitochondrial OxPhos capacity in a $K m t 2 d$-null background that was not apparently compensated by glycolysis, therefore pointing to a general cell bioenergetic deficiency. This conclusion was also supported by direct measurement of the cellular steady-state ATP content, whereby we found a significant $35 \%$ reduction of ATP in $K m t 2 d-K O$ MEF as compared with WT (Figure 1E). The ATP content was sensitive to a short-term exposure of the cells to the $\mathrm{F}_{\mathrm{O}} \mathrm{F}_{1}$-ATP synthase inhibitor oligomycin, providing a measure of the OxPhos contribution and by the difference to that of glycolysis. It can be noted that the ATP contributed by the mitochondrial OxPhos system was largely inhibited in Kmt2d-KO MEF. Conversely, a significant increase of the oligomycin-insensitive ATP content, largely due to glycolysis and other substrate-level phosphorylations, was observed in Kmt2d-KO MEF. Furthermore, we measured in real time the rate of ATP production by utilizing a recently developed and validated Seahorse technology-based protocol. By comparing the rates of $\mathrm{O}_{2}$ consumption and of extracellular $\mathrm{H}^{+}$release, simultaneously measured on the same sample, it is possible to determine the rate of ATP produced by both the OxPhos (mitoATP) and glycolysis (glycoATP) [18]. The results of this analysis, shown in Figure 1E, are in good agreement with the ATP content. Indeed, the rate of ATP produced by the mitochondrial OxPhos was significantly reduced in Kmt2D-KO MEF whereas that produced by glycolysis was increased. It must be highlighted that glycoATP was calculated from the actual release of $\mathrm{H}^{+}$corrected from the contribution of the 
$\mathrm{CO}_{2}$ production and is therefore more reliably linked to the lactate release. We then carried out the same analysis on skin-derived fibroblast cell lines from five Kabuki patients and observed a significant reduction of OCR both under basal and maximal FCCP-stimulated respiration compared to fibroblasts from a healthy subject; additionally, the ATP-linked respiration was inhibited. Basal ECAR was depressed in both Kabuki patients' fibroblasts, whereas the ECAR capacity was decreased in one of them (Figure S1B).
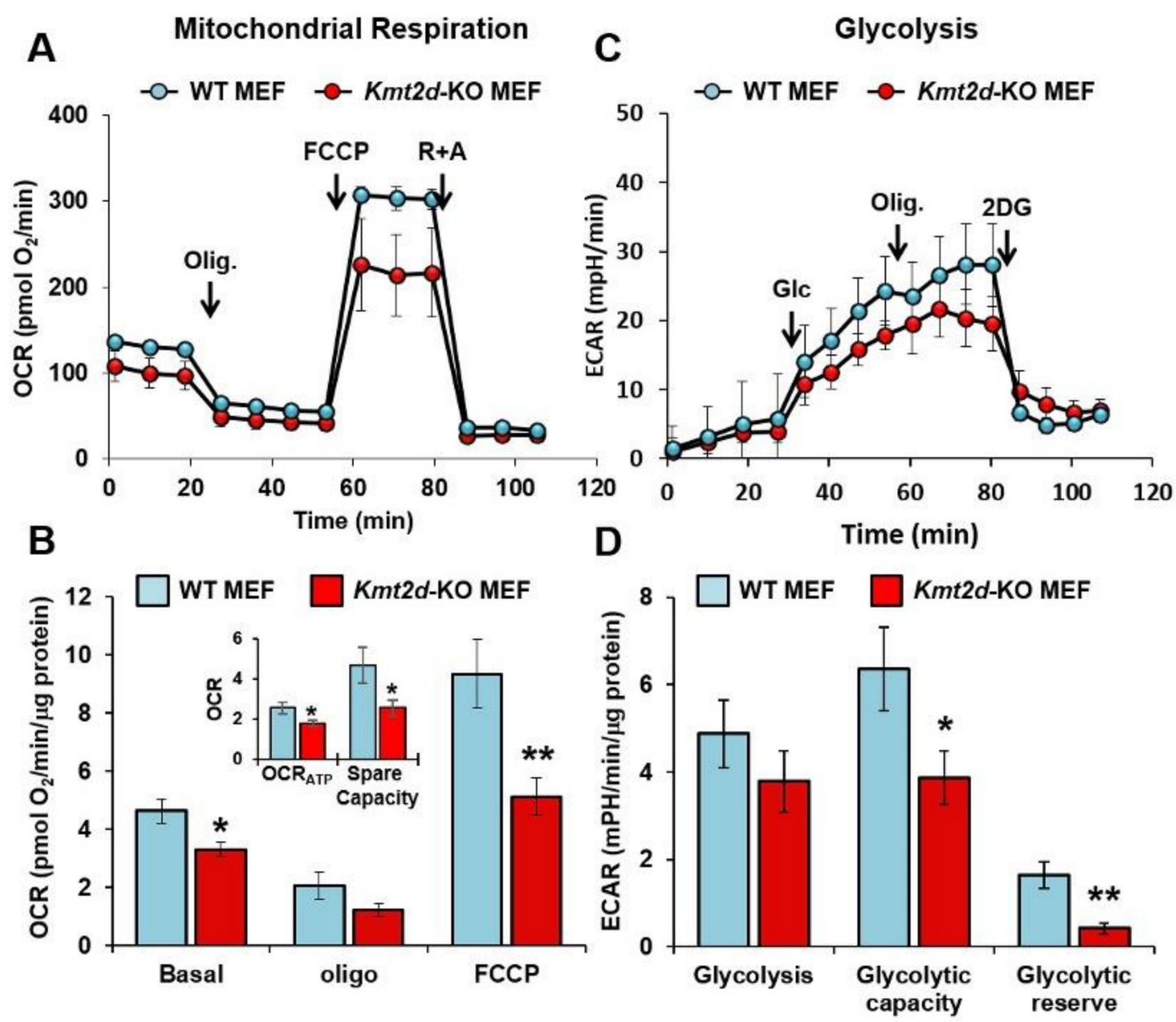

E

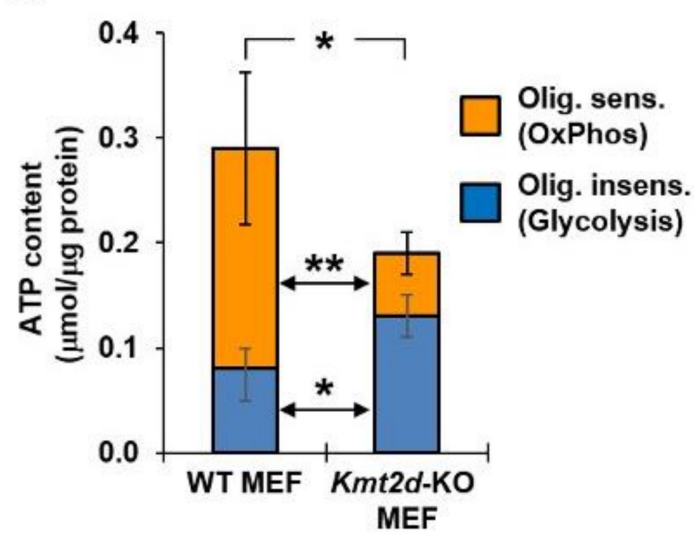

$\mathbf{F}$

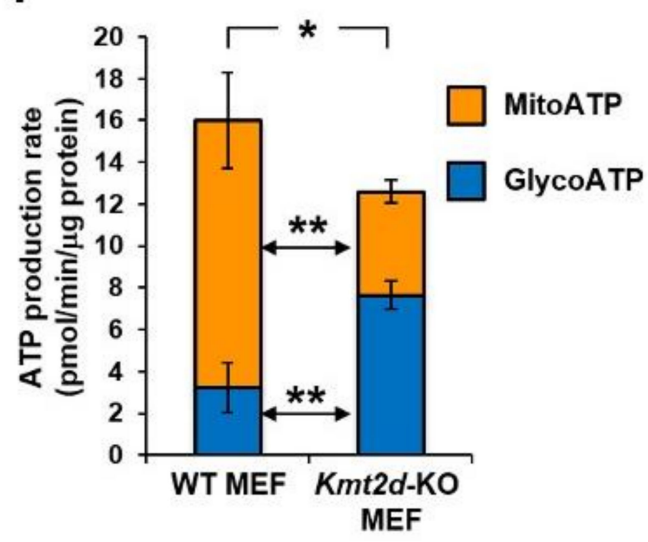

Figure 1. Metabolic fluxes analysis of WT and Kmt2d-KO MEF. Cells were analyzed in microplates by Seahorse XP 96 according to the Mito Stress protocol of the manufacturer. (A) and (C) are representative traces of the oxygen consumption rates (OCRs) and extracellular acidification rates (ECARs), respectively. Where indicated, the following compounds were injected into the assay micro-chambers: oligomycin (Olig.), carbonyl cyanide-4-(trifluoromethoxy)phenylhydrazone (FCCP), rotenone plus antimycin A 
(R+A), for the OCR assay; glucose (Glc), oligomycin, 2-deoxy glucose (2DG), for the ECAR assay. Each data point is mean \pm SD of four technical replicates. $(\mathbf{B}, \mathbf{D})$ are metabolic parameters inferred from the OCR and ECAR assays, respectively. The bars are mean \pm SEM of three independent experiments carried out in quadruplicate under each condition and refer to the R+A-sensitive OCR and 2DG-sensitive ECAR normalized to the protein amount. In (B): Basal, resting OCR; oligo, OCR in the presence of oligomycin; FCCP, OCR under uncoupled condition; in the inset, the ATP-linked OCR (OCR $\left.\mathrm{ATP}_{\mathrm{T}}\right)$ and the respiratory spare capacity (i.e., uncoupled OCR - basal OCR) is also shown. In (D): Glycolysis, ECAR after addition of Glc; Glycolytic capacity, ECAR after addition of oligomycin; Glycolytic reserve, difference between glycolytic reserve and glycolysis. ${ }^{*}, P<0.05$ and ${ }^{* *}, P<0.01$ vs. WT MEF. (E) Cellular ATP steady-state content. ATP was measured as described in the materials and methods in the absence and after $10 \mathrm{~min}$ following addition of $1 \mu \mathrm{M}$ oligomycin; the oligomycin-sensitive and -insensitive ATP content are shown as stacked bars and are the means \pm SEM of three biological replicates; ${ }^{*}, P<0.05$ and ${ }^{* *}, P<0.01$. (F) Rates of ATP production. MEFs were assayed by Seahorse technology utilizing a specific protocol as described in the materials and methods. MitoATP is the mitochondrial OxPhos-linked production of ATP and it was estimated from the OCR corrected for the P/O ratio that was 2.25. GlycoATP is the glycolytic production of ATP and it was estimated from the ECAR following i) conversion of the $\mathrm{pH}$ changes in the absolute amount of $\mathrm{H}^{+}$released (using a calculated buffering power of $1.79 \mathrm{mM}$ $\mathrm{H}^{+} / \mathrm{pH}$ ), and ii) correction from the $\mathrm{CO}_{2}$ release; see the materials and methods and reference therein for details. MitoATP and GlycoATP are shown as stacked bars and are the means \pm SEM of three biological replicates; ${ }^{*}, P<0.05$ and ${ }^{* *}, P<0.01$.

CRISPR-CAS9 was used to generate a knockout KMT2D human skin-derived fibroblast cell line (KMT2D-KO). The metabolite content of the generated fibroblasts clones was investigated by mass spectrometry, and the detected metabolites were clustered according to the metabolic pathways where they are involved. Figure 2 shows the relative levels of intermediates of the glycolysis and tricarboxylic acid (TCA) cycle in KMT2D knockdown fibroblasts when compared with the wild-type isogenic counterpart (Figure 2A,B, respectively). The results attained are consistent with the metabolic flux analysis shown in Figure 1. Indeed, glycolysis appears to be dampened in KMT2D-KO cells at the level of the conversion of D-fructose 1,6-bisphosphate to the trioses phosphates glyceraldehyde-3-phosphate and dihydroxyacetone phosphate, with the levels of both reduced as compared to WT cells. Notably, the detectable intermediates of the TCA cycle were all increased in KMT2D-KO, particularly citrate and succinate. This is consistent with the observed reduction of the mitochondrial respiratory chain and consequent re-oxidation of $\mathrm{NADH}$ and $\mathrm{FADH}_{2}$, which are necessary in the oxido-reduction steps of the TCA cycle.

The defective respiratory activity was further verified by confocal microscopy imaging of the respiration-dependent mitochondrial potential (mt $\Delta \Psi$ ) using the specific fluorescent probe TMRE in Kmt2d-KO cells (Figure 3A). TMRE is a lipophilic cation that accumulates in respiring mitochondria, and this accumulation is driven by the $m t \Delta \Psi$. In $K m t 2 d-K O \mathrm{MEF}$ under resting conditions, the mitochondria-localized probe was significantly reduced, indicating a lower mt $\Delta \Psi$ as compared to its WT counterpart (Figure 3B). Notably, a closer analysis of the functional $\mathrm{mt} \Delta \Psi$-generating mitochondria revealed a more fragmented compartmentalization of the fluorescent signal in cells losing Kmt2d expression. 


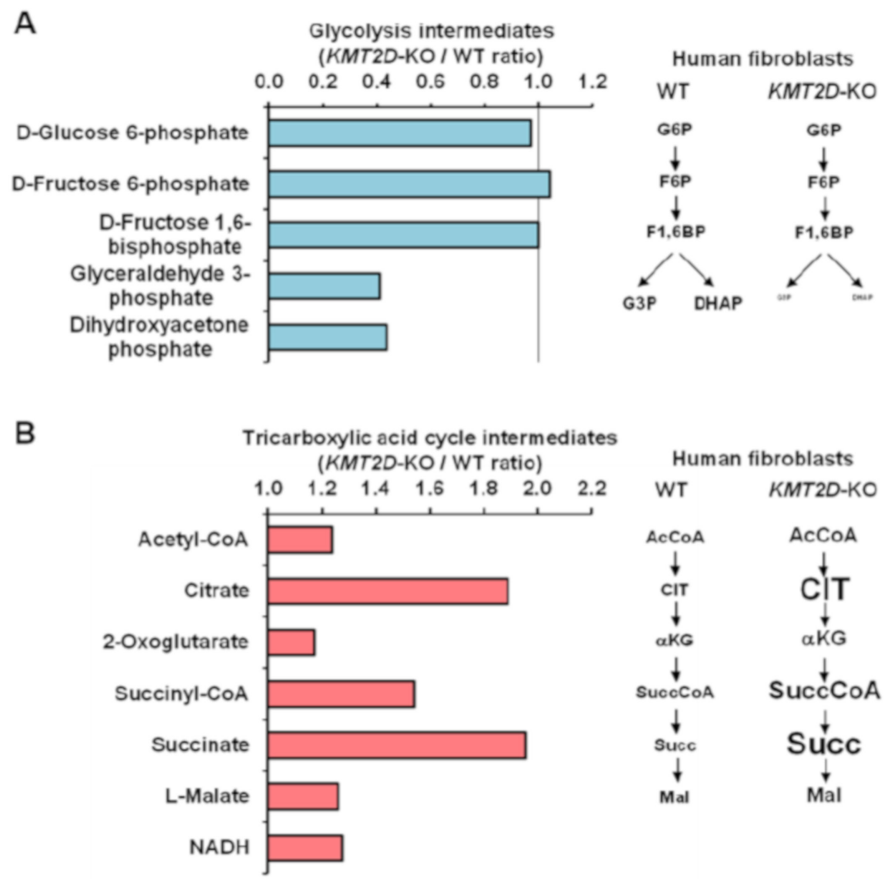

Figure 2. Mass spectrometry analysis of metabolites in wild type (WT) and KMT2D-KO fibroblasts. The histograms in $(\mathbf{A}, \mathbf{B})$ show the relative amounts of intermediate metabolites of glycolysis and the tricarboxylic acid cycle, respectively, in KMT2D-KO fibroblasts as compared with isogenic WT cells. The bars are averages of two biological replicates yielding similar results. The pathways on the right represent the fold change of the indicated metabolites in KMT2D-KO cells as compared with those in WT cells.

A

\section{TMRE (mtAY)}

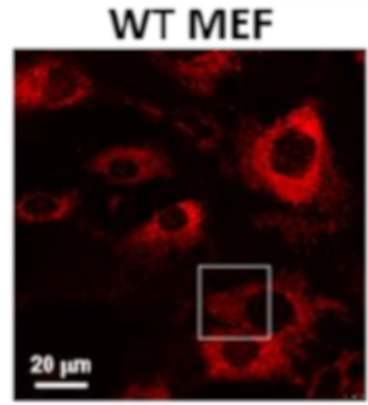

Kmt2d-KO MEF
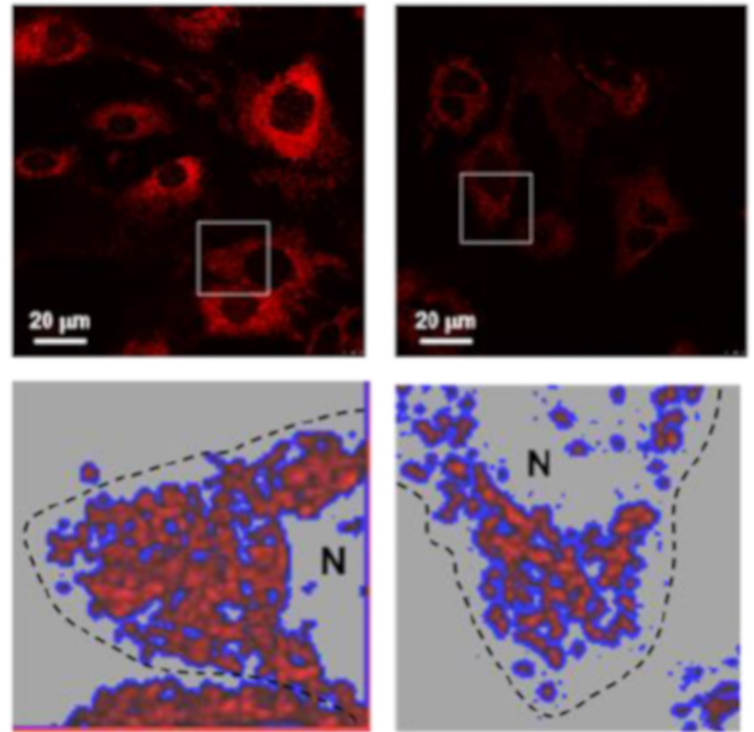

B
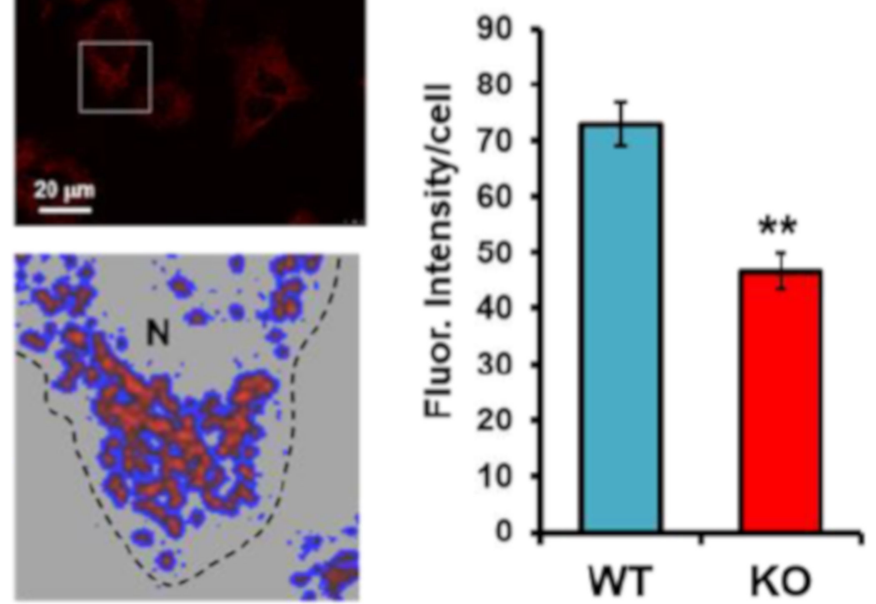

Figure 3. Imaging of the mitochondrial membrane potential in WT and Kmt2d-KO MEF. Cells pre-incubated with TMRE were analyzed by confocal microscopy. (A) Representative images with false-color rendering of digitalized magnifications; N, nucleus. (B) Quantitative analysis of the TMRE fluorescence intensity/cell expressed in arbitrary units; the bars are means \pm SEM of three independent experiments; ${ }^{* *}, P<0.005$. For each condition, at least 10 different optical fields were randomly selected containing 10-15 cells each. See the materials and methods for further details. 


\subsection{Loss of Kmt2d Causes Specific Downregulation of the Respiratory Chain Complexes I and IV}

Because the observed reduced activity of mitochondrial OCR under maximal stimulation could be linked to the content of the respiratory chain complexes, we analyzed the protein level of all complexes (Cs) of the OxPhos system (i.e., Cs I, II, III, IV, V) using a cocktail of antibodies recognizing a specific subunit for each complex. The results attained are illustrated in the immunoblot shown in Figure $4 \mathrm{~A}$ and reveal a selective reduction of $\mathrm{CI}$ (NADH-UQ oxidoreductase/NADH dehydrogenase) and of CIV (cytochrome c oxidase) in Kmt2d-KO MEF as compared with WT MEF. Some reduction was also observed for CII (succinate dehydrogenase), although it did not reach any statistical significance. No difference in the protein expression of $\mathrm{CIII}$ (cytochrome $\mathrm{c}$ reductase) or $\mathrm{CV}\left(\mathrm{H}^{+}-\mathrm{F}_{\mathrm{o}} \mathrm{F}_{1}\right.$ ATP synthase) was observed between the two cell samples. Determination of the specific enzymatic activity of $\mathrm{CI}$ and CIV by spectrophotometric assays confirmed a significant reduction of both complexes in Kmt2d-KO MEF compared to WT MEF (Figure 4B). Conversely, measurement of the citrate synthase activity, which is taken as an indirect marker of the mitochondrial mass, did not show significant changes (Figure 4C).
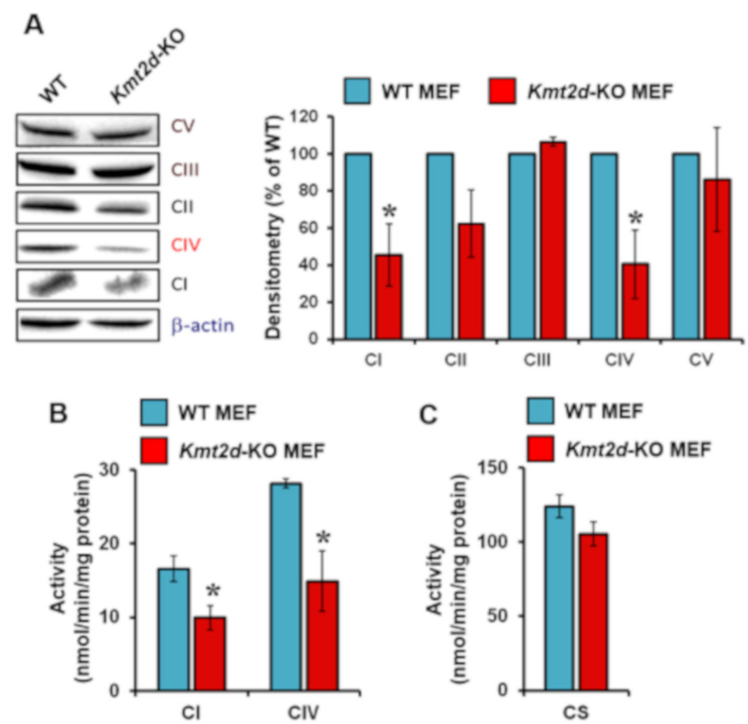

D

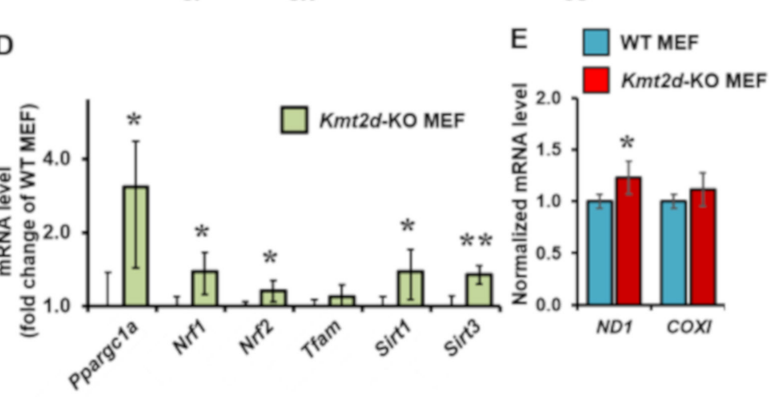

Figure 4. Molecular and functional analysis of the mitochondrial respiratory chain in WT and Kmt2d-KO MEF. (A) Immunoblotting of OxPhos complexes; a representative Western blotting is shown (on the left) with normalized densitometric and statistical analysis (means \pm SEM of $n=4) ;{ }^{*}, P<0.05$ vs. Kmt2d-KO MEF. (B) Enzymatic activity of complex I (CI) and complex IV (CIV); (C) enzymatic activity of citrate synthase; in $(\mathbf{B}, \mathbf{C})$, the activities were carried out by spectrophotometry assay on cell lysate. (D) RT-qPCR analysis of the expression of genes coding for the indicated proteins involved in the mitochondrial biogenesis; the values are fold changes of the transcript in Kmt2d-MEF with respect to WT MEF (means \pm SD of $n=3 ;{ }^{*}, P<0.05 ;{ }^{* *}, P<0.01$ ). (E) RT-qPCR analysis of the expression of mtDNA-coded genes for a subunit of complex I (ND1) and of complex IV (COXI); the values are normalized with respect to WT MEF (means \pm SD of $n=3 ;{ }^{*}, P<0.05$ ). 
To verify if the reduced content of the respiratory chain complexes I and IV was due to defective biogenesis, we measured the expression level of the major transcription factors controlling the bi-genomic expression of the respiratory chain complexes by RT-qPCR. The expression of Pgc1a, which codes for the master transcription factor peroxisome proliferator-activated receptor gamma coactivator 1-alpha (PGC-1 $\alpha$ ), was three-fold higher in Kmt2d-KO MEF compared to WT MEF (Figure 4D). There was also a slight significant increase in the expression of the nuclear respiratory transcription factors $1 / 2$ (Nrf1/2), the histone/protein deacetylases sirtuins $1 / 3$ (Sirt1/3). The expression of the mitochondrial transcription factor A (Tfam), which is controlled by PGC- $1 \alpha$, was also increased, though not reaching statistical significance. Consistent with the modest effect on Tfam expression, which controls transcription of mtDNA genes, the expression levels of $N d 1$ and CoxI, both coded by the $\mathrm{mt} D N A$, were slightly increased, with statistical significance only for $N d 1$ (Figure 4E). This counterintuitive result would suggest an adaptive response in $K m t 2 d-\mathrm{KO}$ cells to the OxPhos dampening, which, however, did not result in a compensative outcome.

\subsection{Loss of Kmt2d Causes Unbalance in the Reactive Oxygen Species Homeostasis}

Previous studies have shown that deregulation of the mitochondrial respiratory chain often leads to enhanced generation of reactive oxygen species (ROS) [22]. Therefore, we assessed the intracellular redox state by imaging $\mathrm{K} m+2 \mathrm{~d}-\mathrm{KO} \mathrm{MEF}$ in the presence of ROS-sensitive probes. Although the probe dichlorofluorescein (DCF) responds to a variety of reactive species, it displays some selectivity with peroxides [23]. Comparative confocal microscopy imaging and signal quantification revealed a significantly higher level of DCF-related fluorescence in Kmt2d-KO MEF (Figure 5A). Consistently, we observed an increase of fluorescent signal in Kabuki patients' fibroblasts when compared to control fibroblasts (Supplementary Material Figure S2). Finally, Figure 5B shows the result attained using the probe MitoSox, which specifically detects the intramitochondrial superoxide anion species $\left(\mathrm{O}_{2}{ }^{\bullet-}\right)$. No difference in the MitoSox-related fluorescence signal was observed between WT MEF and Kmt2d-KO MEF. These findings prompted us to check the expression of genes coding for major antioxidant enzymes by using RT-qPCR (Figure 5C). Most of the selected antioxidant genes in Kmt2d-KO MEF were upregulated, with this increase reaching statistical significance for Gsr (coding for glutathione reductase), $\operatorname{Prd} x 5$ (mitochondrial peroxiredoxin-5), Sod1 (cytoplasmic $\mathrm{Cu}-\mathrm{Zn}$ superoxide dismutase-1), and Sod2 (mitochondrial Mn superoxide dismutase-2). 

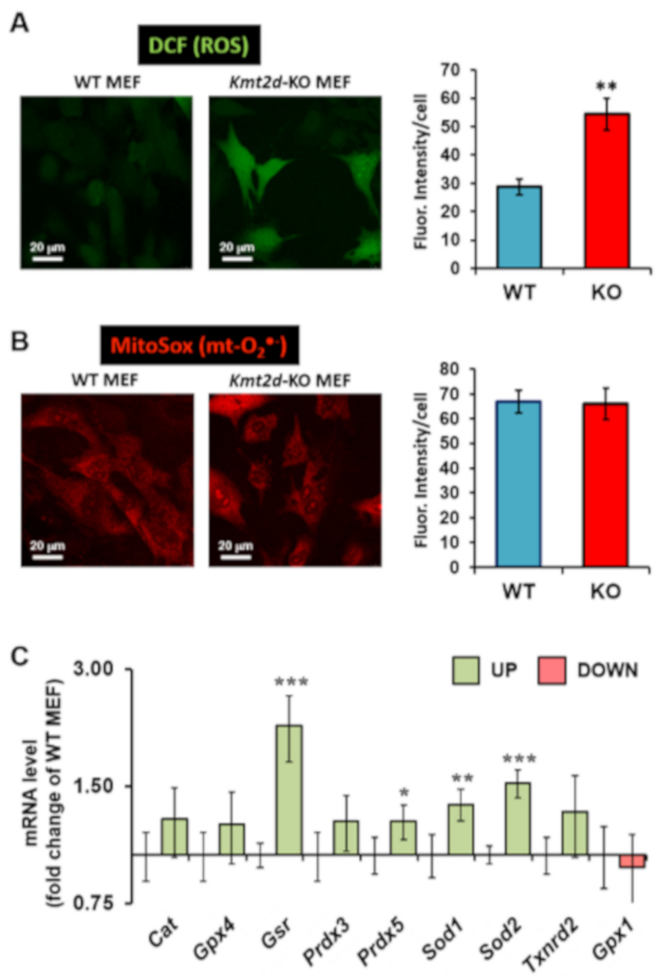

Figure 5. Assessment of the redox homeostasis in Kmt2d-KO and WT MEF. (A,B) show the confocal microscopy analysis of cells loaded with the redox-sensitive fluorescent probes DCF and MitoSox, respectively. The panels on the left are representative images, and the histograms on the right are quantification of the fluorescent signal/cell. The bars are means \pm SEM of three independent experiments; **, $P<0.005$. For each condition, at least 10 different optical fields were randomly selected containing 10-15 cells each. (C) RT-qPCR analysis of gene expression for the indicated proteins involved in the antioxidant defense; the values are fold changes of the transcript in Kmt2d-KO MEF with respect to WT MEF (means $\pm \mathrm{SD}$ of $n=3 ;{ }^{*}, P<0.05 ;{ }^{* *}, P<0.05 ;{ }^{* *}, P<0.001$ ).

\subsection{Loss of Kmt2d Changes the Glucose- and Fatty Acid-Related Metabolic Flexibility}

Next, we sought to verify whether, in addition to the impaired mitochondrial respiratory chain, the loss of KMT2D function influences the reliance of the aerobic metabolism on specific oxidizable substrates. In cultured cells, the major respiratory substrates are glucose (Glc), fatty acids (FAs), and glutamine (Gln). Thus, to evaluate the link between KMT2D and these major respiratory substrates, we treated Kmt2d-KO MEF with a cocktail of the following drugs: UK5099, which inhibits mitochondrial pyruvate carrier (MPC); etomoxir, which inhibits carnitine palmitoyltransferase-1 (CPT-1); and BPTS, which inhibits glutaminase (Figure 6A). Figure 6B shows that this treatment resulted in a slightly higher reliance of the basal OCR on these three metabolic substrates in Kmt2d-KO MEF as compared with WT MEF. Other unidentified substrates, however, may contribute to the respiratory activity in both WT and Kmt2d-KO MEFs. 
A

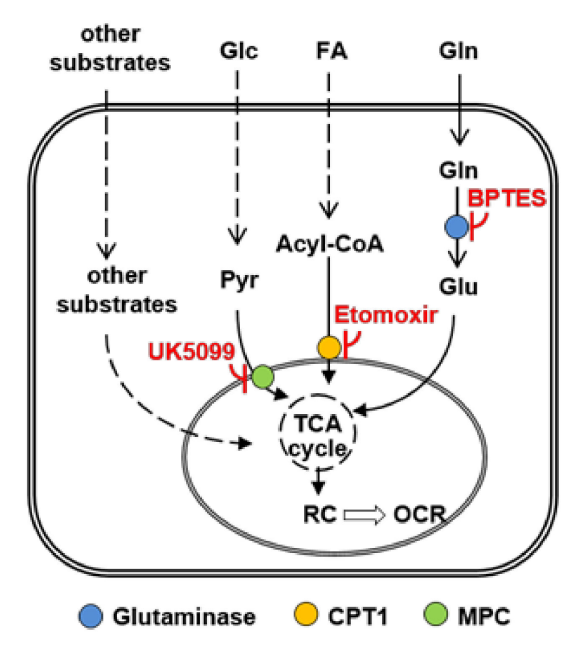

B

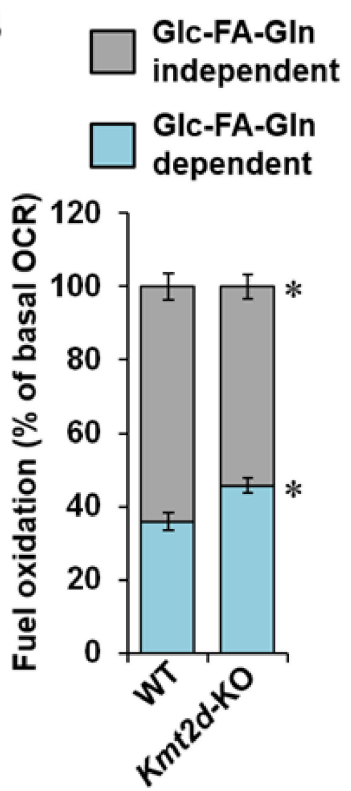

Figure 6. Measurement of the reliance on respiratory substrates in $\mathrm{K} m \mathrm{t} 2 \mathrm{~d}$-KO and WT-MEF. Resting OCRs were assessed in cells by a Seahorse Analyser in the absence and in the presence of a cocktail of inhibitors (UK5099, etomoxir, and BPTS). (A) The targeted pathways of the inhibitors are shown; CPT1, carnitine palmitoyl transferase 1; MPC, mitochondrial pyruvate carrier; Glc, glucose; FA, long-chain fatty acids; Gln, glutamine; Glu, glutamate; Pyr, pyruvate. (B) Histogram showing the Glc/FA/Gln-dependent and Glc/FA/Gln-independent OCRs expressed as percentage of the rotenone + antymicin A-sensitive OCR; means \pm SEM of $n=4$ independent biological replicates in triplicate; *, $P<0.05$.

To better characterize the specific contributions of Glc, FA, and Gln to mitochondrial respiration, the endogenous OCR was assessed by mitochondrial fuels screening (MFS) using separate inhibitors of specific target pathways. From the MFS analysis, it is possible to estimate the dependency and the capacity of a given pathway and, by the difference between the two, its flexibility. Dependency indicates the relative amount of basal mitochondrial oxidation from a single fuel that cannot be compensated through oxidation of the other two fuels. Capacity is the relative ability of a cell to oxidize a specific fuel in the basal state when oxidation of the other two fuels is blocked. Flexibility is the difference between capacity and dependency. It indicates the relative ability of a cell in the basal state to switch or compensate mitochondrial oxidation from one fuel to another. Figure 7A,B show the representative oxymetric traces attained by MFS assay for FA oxidation in WT MEF and Kmt2d-KO MEF, respectively. Similar analysis of the Glc and Gln pathways revealed observable differences between $K m t 2 d-K O$ and WT MEF (Figure 7C). In particular, in WT MEF, the OCR dependencies from all three substrates almost coincided with their capacities but with a small but significantly higher reliance on fatty acid oxidation (FAO). In Kmt2d-KO MEF, the dependency on Glc was lower compared to that of FA and Gln and compared to the Glc dependency in WT MEF. However, Kmt2d-KO MEF exhibited larger flexibility of the Glc and FA pathways. Because of this, their Glc capacity was comparable to that of WT MEF, and the FAO capacity was even larger. 
A
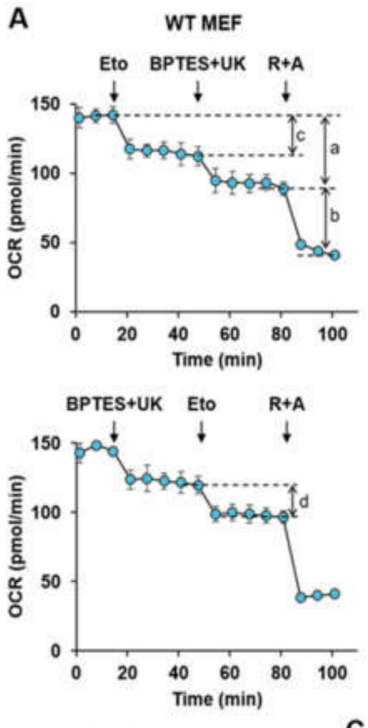

$\mathrm{a}=$ OCR GIC/FA/GIn-dependent $b=$ OCR GIE/FA/GIn-independen $c=F A$ Dependency $d=$ FA Capacity d. $c=$ FA Flexibility

C
B Kmt2d-KO MEF
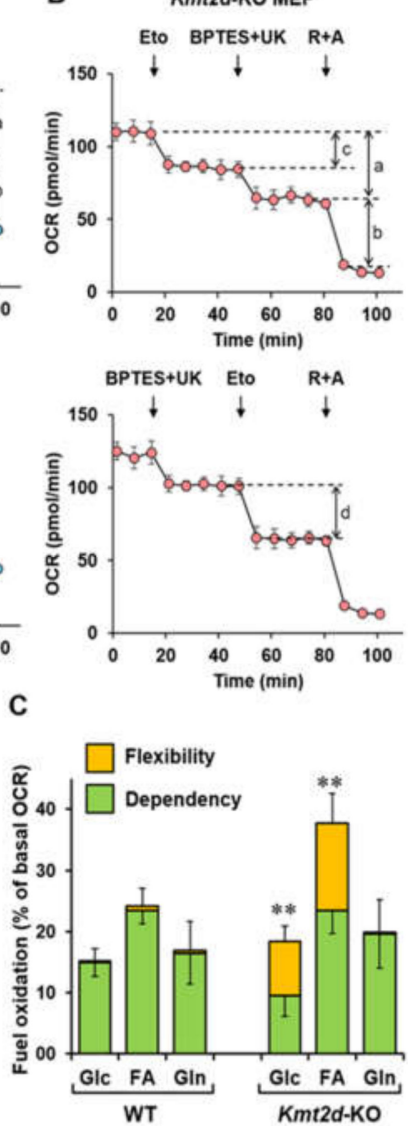

Figure 7. Mitofuel assay of Kmt2d-KO and WT MEF. Cells were assayed by the Seahorse Mito-Fuel kit as detailed in the materials and methods. (A) and (B) representative oximetric traces to assess the contribution of long-chain fatty acids (FA)s to the resting OCR. Where indicated, inhibitors of targeted pathways (see Figure 6A) were added: eto, etomoxir; BPTS + UK (5099); R+A, rotenone + antymicin A. The OCR values were utilized to extract the specific dependency, capacity, and flexibility on FA oxidation. The Glc/FA/Gln-dependent and Glc/FA/Gln-independent respiration is also shown and detailed in the legend. (C) Histograms showing the relative dependency and flexibility in the utilization of glucose/pyruvate (Glc), long-chain fatty acids (FAs), and glutamine (Gln) in cell respiration; the bars are mean relative values \pm SEM of $n=4$ independent biological replicates in triplicate; ${ }^{* *}, P<0.01$.

The higher FAO flexibility in Kmt2d-KO MEF was further verified by measuring the etomoxir-sensitive OCR in the presence of exogenous palmitate. Figure 8 shows that no significant difference in WT MEF OCR was observed, irrespective of the presence of FA palmitate. Conversely, the OCR in Kmt2d-KO MEF was lower compared to the OCR in WT MEF in the absence of FA, and it was stimulated in the presence of FA palmitate. Additionally, the OCR in Kmt2d-KO MEF was much more sensitive to the FAO inhibitor etomoxir. Altogether, these results suggest that, in a KMT2D-null background, a rewiring of the metabolic phenotype occurs. 


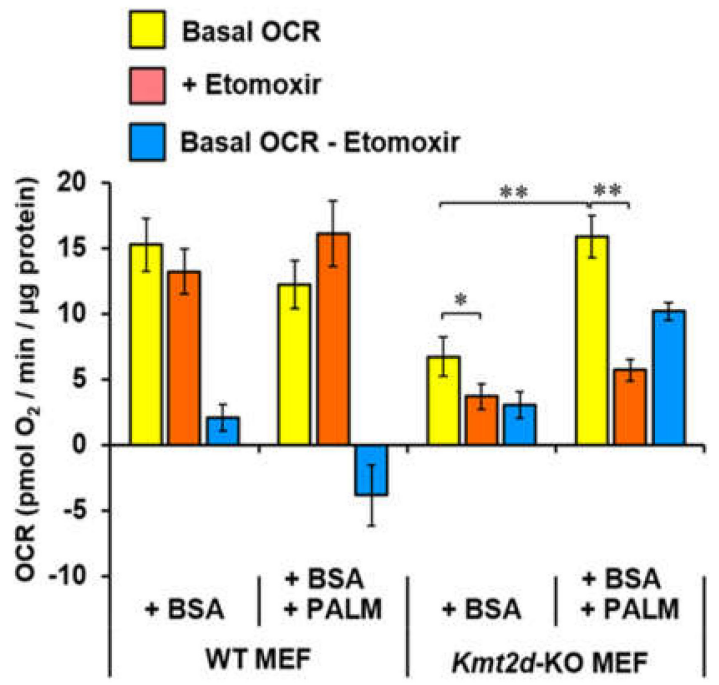

Figure 8. Fatty acids-related respiratory activity of Kmt2d-KO and WT MEF. Cells were assessed for resting OCR. Medium was supplemented with bovine serum albumin (+BSA) or with BSA + palmitate (+BSA +PALM); etomoxir was added to inhibit long-chain fatty acid oxidation. Bars are means \pm SEM of $n=3$ independent biological replicates in triplicate; ${ }^{*}, P<0.05 ;{ }^{* *}, P<0.01$.

\section{Discussion}

The link between cellular metabolism and the epigenetic landscape is emerging as an important determinant of cell phenotype $[1,14,24]$. This notion is highlighted by increasing evidence pointing to the impact of physiological modulation or pathological alteration of given metabolic pathways on reversible modifications of DNA and histones. For instance, methylation of DNA cytosines and histone lysines, which are among the most common epigenetic modifications, all rely on S-adenosylmethionine, despite the number of different methylases involved. The regeneration of S-adenosylmethionine mainly depends on the folate cycle, which requires NADH and serine. On the other hand, demethylation by some classes of demethylating dioxygenases is tightly dependent on the availability of 2-oxoglutarate, an intermediate of the TCA cycle. Similarly, acetylation of histones requires available nuclear acetyl-CoA, which is largely generated by mitochondrial-located pathways. Conversely, histone deacetylation by sirtuins is NAD+ dependent.

Here, we investigated the impact of KMT2D on the bioenergetic cell metabolism, choosing the mitochondria as the target for our investigations. Using the Seahorse methodology, we assessed both the mitochondrial respiratory parameters and the glycolytic flux and their dependence on specific oxidizable substrates in Kmt2d-KO MEF.

The results attained clearly show that the resting mitochondrial respiratory activity supported by endogenous substrates was significantly inhibited in a KMT2D-null context. Since the respiratory activity in the presence of a selective inhibitor of FoF1-ATP-synthase was scarcely affected, we can conclude that the relative portion of the OCR that translates to ATP production is negatively regulated by KMT2D. This was also confirmed by the significant reduction of the mitochondrial membrane potential, which is a measure of the respiratory chain to couple chemiosmotically their proton pumping activity to the synthesis of ATP. Notably, analysis of the glycolytic flux did not show any compensatory mechanism, which confirms the bioenergetic deficit in KMT2D-deficient cells.

The observed reduced maximal OCR in $K m t 2 d-K O$ MEF suggested a potential reduction in the content of respiratory chain complexes. However, intriguingly, immunoblotting analysis confirmed a reduced expression of only complexes I and IV. Consistent with these findings, the enzymatic activities of complex I and IV were also reduced. In contrast, the enzymatic activity of citrate synthase was unchanged in Kmt2d-KO MEF. Complexes I and IV are both committed steps in the respiratory chain electron transfer, and their stalling may cause electron slippage to molecular oxygen, generating reactive oxygen species [22]. Consistently, there was a significant increase in cellular peroxides in Kmt2d-KO MEF. 
The Kmt2d-KO cells seemed to partially compensate for this mitochondrial dysfunction through the overexpression of antioxidant enzymes and transcription factors involved in mitochondrial biogenesis. Nevertheless, these adapting responses did not succeed in preventing the mitochondrial alterations.

Impaired transfer of reducing equivalents to molecular oxygen is also consistent with the observed accumulation of intermediates of the TCA cycle, which depends on the re-oxidation of NADH. Moreover, a blockage of the glycolytic flux was also observed at the level of the aldolase-mediated conversion of fructose 1,6-bisphosphate to the triosephosphates.

In addition to the defective respiratory chain, analysis of the contribution of different substrates to the OCR demonstrated a greater flexibility of $K m+2 d-K O$ MEF in the utilization of pyruvate and long-chain FAs. Moreover, a relative higher capacity of FA oxidation was observed in Kmt2d-KO MEF, in part due to its heightened flexibility for FA oxidation. This conclusion is also supported by the observed changes in intermediates of the TCA cycle. Indeed, the accumulation of citrate in Kmt2d-KO MEF, wherein FA oxidation is favored, is most likely because this metabolite is normally utilized as a precursor in FA biosynthesis. Succinate accumulation may be due to enhanced production of succinyl-CoA, derived from the degradation of several amino acids and odd-chain FAs. Oxidation of succinate is also hampered by the lower availability of NAD+, which is due to lower electron transfer activity of the respiratory chain. Consequently, if oxidation of malate to oxaloacetate is hampered, this causes an accumulation of intermediates that convert back to succinate given that all reactions in this part of the TCA cycle are reversible. To note, succinate cannot be converted into succinyl-CoA because the reaction is thermodynamically unfavored, thus contributing to the accumulation of succinate.

Altogether, these results indicate that KMT2D controls the aerobic metabolism by maintaining the proper balance of enzymatic respiratory chain complexes (see the schematic drawing in Figure 9). In the last decade, convincing evidence has indicated that the respiratory chain complexes I, III, and IV are assembled in situ in supercomplexes with defined stoichiometry [25]. This would maximize the efficiency of electron transfer from NADH to dioxygen while limiting the production of reactive species [26]. Each of the three multi-subunit complexes are bi-genomic as some of their subunits are encoded by nuclear genes while others are encoded by mitochondrial DNA [27]. These two transcriptional machineries are regulated by different transcription factors. One of the best characterized transcription factors is PGC- $1 \alpha$, a master regulator of mitochondrial biogenesis [28,29]. This protein interacts with nuclear receptor PPAR-g, thereby allowing its interaction with multiple transcription factors $[28,30]$.

Probing an already published extensive Chip-seq dataset for Kmt2d target genes [5], it was possible to test the involvement of them in the defective functions investigated in this study. As expected, many of the metabolic genes (involved in glycolysis and the TCA cycle) were targets of Kmt2d as well as genes coding for the antioxidant armory. Concerning the genes directly involved in the mitochondrial OxPhos system, with the obvious exclusion of the mitochondrial DNA genes, several nuclear genes encoding for subunits of CII, CIII, CIV, and CV were direct Kmt2d targets. Even if no evidence was found about $\mathrm{CI}$, its decreased expression might be indirectly linked to the failure to stabilize respiratory CI-containing supercomplexes when CxIII and CIV are defective [31,32]. All this supports the deregulated oxidative metabolism reported in our study in a KMT2D-deficient context. 

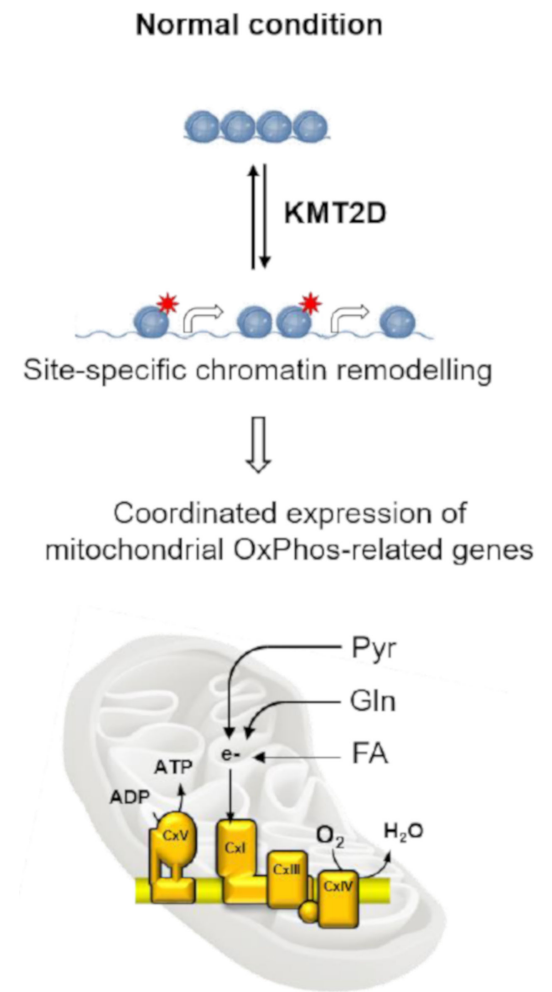

Kabuki

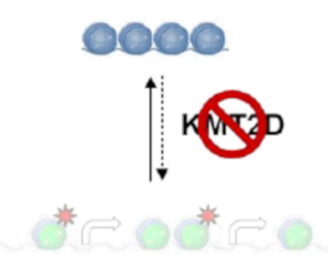

$\sqrt{6}$

Deregulated expression of mitochondrial OxPhos-related genes

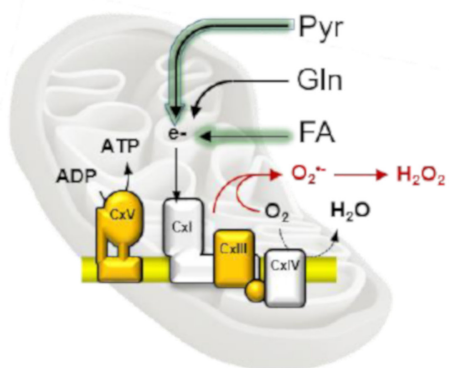

Figure 9. Schematic overview of the effects elicited by KMT2D on the aerobic metabolism as inferred from this study. The normal condition is shown on the left, with KMT2D-mediated chromatin remodeling controlling the coordinated expression of mitochondrial OxPhos-related genes. Alterations in the KMT2D-null context as in the Kabuki syndrome are shown on the right. Impairments include decreased expression and activity of the respiratory chain complexes I and IV with reduced oxygen consumption and the generation of reactive oxygen species. Altered flexibility in the oxidative utilization of pyruvate (Pyr) and long-chain fatty acids (FAs) is also shown as shadowed arrows; Gln, glutamine. See the discussion for further explanation.

Consistent with the alteration of energetic metabolism in a KMT2D-null background, a number of lines of evidence are consistent with the data reported here. It has been reported that heterozygous $K M T 2 D^{+/-}$mice display an altered metabolic phenotype hallmarked by enhanced glucose tolerance, insulin sensitivity, and a higher serum bile acid level [12], as well as resistance to high-fat diet-induced hepatic steatosis [13]. KMT2D was consistently found to be recruited at enhancer sites by PPAR- $\gamma$, where it acted as a coactivator [33]. Thus, it was found to promote the transactivation of selected genes involved in rewiring lipid and cholesterol metabolism [13]. However, as mentioned, PPAR- $\gamma$ is also involved in the homeostasis of mitochondrial function in tandem with its coactivator PGC-1 $\alpha$ [30]. P53 is a major transcription factor found to be deregulated in a KMT2D-KO context [34]. In addition to its well-recognized function as a tumor suppressor due to its role in nuclear DNA repair, P53 is also involved in maintenance of the mitochondrial genome [35]. Indeed, the transcription factor A mitochondrial (TFAM), a gene necessary for both transcription and replication of mtDNA, has been reported to be a transcriptional target of P53 [36]. Notably, P53 also targets synthesis of cytochrome c oxidase 2 (SCO2), a factor involved in the assembly of the cytochrome c oxidase complex, thereby regulating mitochondrial respiration [37]. Using RNA-Seq analysis, it was revealed that KMT2D is, along with KMT2C, a key epigenetic regulator of the circadian clock and functions as a coactivator of the circadian transcription factors retinoid-related orphan receptor (ROR)- $\alpha$ and $-\gamma$ [12]. Recent evidence supports a tight interplay between cell-autonomous biological clocks and metabolism, particularly with mitochondrial respiratory function [38,39]. In pancreatic carcinogenesis, transcriptional repression of KMT2D was found to be double-site CpG methylation dependent and was linked to metabolic reprogramming. In particular, the loss of H3K4me3 was associated 
with altered expression of the glucose transporter SLC2A3, a decreased OCR/ECAR ratio, and deregulation of FA oxidation and the lipidomic profile [40]. Loss of DPY30, a component of the ASCOM complex and facilitator of genome-wide KMT2D-mediated H3K4 methylation [41], in hematopoietic stem/progenitor cells (HSPCs) impairs the transition between pluripotent and differentiated states [42]. This was caused by impaired energy metabolism, including both glycolytic and mitochondrial pathways [43]. In a mouse model of KMT2D deficiency, early maturation of neuronal stem/progenitor cells was reported, along with strong perturbation of hypoxia-responsive metabolism pathways encompassing glycolysis, mitochondrial OxPhos, and mitochondrial transcription [44]. In the $K m t 2 d^{+/ b G e o}$ mouse model of Kabuki, a beneficial effect of a ketogenic diet intervention was demonstrated in rescuing neurogenesis defects and hippocampal abnormalities [45]. This effect was traced back to an elevated $\beta$-hydroxybutyrate/acetoacetate ratio specifically observed in $K m+2 d^{+/ b G e o}$ mice. Mechanistically, it was proposed that the accumulation of $\beta$-hydroxybutyrate, an inhibitor of the histone deacetylase, counteracted the defective histone methyl transferase activity in $K m+2 d^{+/ b G e o}$ mice. Ketone bodies formed following a ketogenic diet are catabolic fuels serving as respiratory substrates of the mitochondrial respiratory chain. A defective respiratory chain function with stalled $\mathrm{NADH}$ oxidation, such as that found in our study with the Kmt2d-KO model, might well account for both the accumulation of upstream substrates, such as ketone bodies, and their conversion in $\beta$-hydroxybutyrate.

\section{Conclusions}

In this study, we provided experimental evidence for a formerly unappreciated specific function of KMT2D in controlling aerobic metabolism. However, the mechanistic hints on how a KMT2D-null context affects bioenergetic metabolism remain unsolved. Although further studies are needed to understand if the reported effects are primarily linked to the KMT2D loss of function or mediated by secondary compensatory mechanisms, our findings may shed light on the metabolic alterations contributing to these complex disease phenotypes.

Supplementary Materials: The following are available online at http://www.mdpi.com/2073-4409/9/7/1685/s1, Figure S1. Metabolic fluxes analysis in normal and Kabuki syndrome-affected patients' fibroblasts, as described in the legend of Figure 1. The mutations in KMT2D carried on by each Kabuki patient are indicated in (A). (A) and (B) are metabolic parameters inferred from the OCR and ECAR assays, respectively. The bars are means SEM of three independent experiments carried out in triplicate under each condition. (C) and (D) are OCR- and ECAR-related parameters resulting from the averaging of the four Control and five Kabuki fibroblasts; ${ }^{*}, P<0.05$ vs control fibroblasts. In the table below further features of the patients' fibroblasts are indicated. Figure S2. Measurement of the intracellular ROS in normal and Kabuki syndrome-affected patients' fibroblasts. DCF-loaded fibroblasts were analysed by confocal microscopy as in Figure 5A and the mean fluorescence intensity/cell of the probe was estimated as described in Materials and Methods. The bars are mean SEM of three independent experiments for each sample; ${ }^{*}, P<0.05$ and ${ }^{* * *}, P<0.0005$ vs normal (CTRL) fibroblasts. Table S1. OFF Target prediction for gRNA used for KMT2D KO of human patient's cell lines. (http://www.rgenome.net/cas-offinder/).

Author Contributions: Conceptualization, N.C. and G.M.; methodology, C.P. (Consiglia Pacelli), P.P., N.C., G.M.; formal analysis, C.P. (Consiglia Pacelli), P.P., N.C.; investigation, experimental design and realization, C.P. (Consiglia Pacelli), I.A., N.M., G.M.S., C.P. (Claudia Piccoli), A.A., G.P.; data curation, C.P. (Consiglia Pacelli), N.C.; N.C.; writing-original draft preparation; writing—review and editing, C.P. (Consiglia Pacelli), N.C., G.M., K.G., J-E.L.; supervision, N.C., G.M.; funding acquisition, G.M., C.P. (Consiglia Pacelli), N.C.; preparation and provision of MEF cell lines, J.-E.L., K.G. All authors have read and agreed to the published version of the manuscript.

Funding: This research was funded by: "Interveento cofinanziato dal Fondo di Sviluppo e Coesione 2007-2013-APQ Ricerca Regione Puglia Programma regionale a sostegno della specializzazione intelligente e della sostenibilità sociale ed ambientale-FutureInResearch" H6SH8W9, Italy to C.P. (Consiglia Pacelli); Telethon Italy (grant \#GGP13231), the Italian Ministry of Health, Jerome Lejeune Foundation, and Daunia Plast.

Acknowledgments: We thanks Kaitlin McKernan for the manuscript proofreading; the authors are grateful to AISK (Associazione Italiana Sindrome Kabuki) for continue inspiration.

Conflicts of Interest: The authors declare no conflict of interest. 


\section{References}

1. Reid, M.A.; Dai, Z.; Locasale, J.W. The impact of cellular metabolism on chromatin dynamics and epigenetics. Nat. Cell Biol. 2017, 19, 1298-1306. [CrossRef] [PubMed]

2. Froimchuk, E.; Jang, Y.; Ge, K. Histone H3 lysine 4 methyltransferase KMT2D. Gene 2017, 627, $337-342$. [CrossRef] [PubMed]

3. Guo, C.; Chen, L.H.; Huang, Y.; Chang, C.C.; Wang, P.; Pirozzi, C.J.; Qin, X.; Bao, X.; Greer, P.K.; McLendon, R.E.; et al. KMT2D maintains neoplastic cell proliferation and global histone H3 lysine 4 monomethylation. Oncotarget 2013, 4, 2144-2153. [CrossRef] [PubMed]

4. Hu, D.; Gao, X.; Morgan, M.A.; Herz, H.M.; Smith, E.R.; Shilatifard, A. The MLL3/MLL4 branches of the COMPASS family function as major histone H3K4 monomethylases at enhancers. Mol. Cell Biol. 2013, 33, 4745-4754. [CrossRef]

5. Lee, J.E.; Wang, C.; Xu, S.; Cho, Y.W.; Wang, L.; Feng, X.; Baldridge, A.; Sartorelli, V.; Zhuang, L.; Peng, W.; et al. H3K4 mono- and di-methyltransferase MLL4 is required for enhancer activation during cell differentiation. Elife (Cambridge) 2013, 2, e01503.

6. Squeo, G.M.; Augello, B.; Massa, V.; Milani, D.; Colombo, E.A.; Mazza, T.; Castellana, S.; Piccione, M.; Maitz, S.; Petracca, A.; et al. Customised next-generation sequencing multigene panel to screen a large cohort of individuals with chromatin-related disorder. J. Med. Genet. 2020. [CrossRef]

7. Adam, M.P.; Hudgins, L.; Hannibal, M. Kabuki Syndrome. 1993.

8. Wang, Y.R.; Xu, N.X.; Wang, J.; Wang, X.M. Kabuki syndrome: Review of the clinical features, diagnosis and epigenetic mechanisms. World J. Pediatr. 2019, 15, 528-535. [CrossRef]

9. Lederer, D.; Grisart, B.; Digilio, M.C.; Benoit, V.; Crespin, M.; Ghariani, S.C.; Maystadt, I.; Dallapiccola, B.; Verellen-Dumoulin, C. Deletion of KDM6A, a Histone Demethylase Interacting with MLL2, in Three Patients with Kabuki Syndrome. Am. J. Hum. Genet. 2012, 90, 119-124. [CrossRef]

10. Kaikkonen, M.U.; Spann, N.J.; Heinz, S.; Romanoski, C.E.; Allison, K.A.; Stender, J.D.; Chun, H.B.; Tough, D.F.; Prinjha, R.K.; Benner, C.; et al. Remodeling of the enhancer landscape during macrophage activation is coupled to enhancer transcription. Mol. Cell 2013, 51, 310-325. [CrossRef]

11. Ortega-Molina, A.; Boss, I.W.; Canela, A.; Pan, H.; Jiang, Y.; Zhao, C.; Jiang, M.; Hu, D.; Agirre, X.; Niesvizky, I.; et al. The histone lysine methyltransferase KMT2D sustains a gene expression program that represses B cell lymphoma development. Nat. Med. 2015, 21, 1199-1208. [CrossRef]

12. Kim, D.H.; Rhee, J.C.; Yeo, S.; Shen, R.; Lee, S.K.; Lee, J.W.; Lee, S. Crucial roles of mixed-lineage leukemia 3 and 4 as epigenetic switches of the hepatic circadian clock controlling bile acid homeostasis in mice. Hepatology 2015, 61, 1012-1023. [CrossRef] [PubMed]

13. Kim, D.H.; Kim, J.; Kwon, J.S.; Sandhu, J.; Tontonoz, P.; Lee, S.K.; Lee, S.; Lee, J.W. Critical Roles of the Histone Methyltransferase MLL4/KMT2D in Murine Hepatic Steatosis Directed by ABL1 and PPARgamma2. Cell Rep. 2016, 17, 1671-1682. [CrossRef] [PubMed]

14. Matilainen, O.; Quiros, P.M.; Auwerx, J. Mitochondria and Epigenetics - Crosstalk in Homeostasis and Stress. Trends Cell Biol. 2017, 27, 453-463. [CrossRef]

15. Mora, M.; Angelini, C.; Bignami, F.; Bodin, A.M.; Crimi, M.; Di Donato, J.H.; Felice, A.; Jaeger, C.; Karcagi, V.; LeCam, Y.; et al. The EuroBioBank Network: 10 years of hands-on experience of collaborative, transnational biobanking for rare diseases. Eur. J. Hum. Genet. 2015, 23, 1116-1123. [CrossRef] [PubMed]

16. Bae, S.; Park, J.; Kim, J.S. Cas-OFFinder: A fast and versatile algorithm that searches for potential off-target sites of Cas9 RNA-guided endonucleases. Bioinformatics 2014, 30, 1473-1475. [CrossRef]

17. McCloskey, D.; Ubhi, B.K. Quantitative and Qualitative Metabolomics for the Investigation of the Intracellular Matabolism. SCIEX TECH Note 2014, 1-11.

18. Mookerjee, S.A.; Gerencser, A.A.; Nicholls, D.G.; Brand, M.D. Quantifying intracellular rates of glycolytic and oxidative ATP production and consumption using extracellular flux measurements. J. Biol. Chem. 2017, 292, 7189-7207. [CrossRef]

19. Cela, O.; Piccoli, C.; Scrima, R.; Quarato, G.; Marolla, A.; Cinnella, G.; Dambrosio, M.; Capitanio, N. Bupivacaine uncouples the mitochondrial oxidative phosphorylation, inhibits respiratory chain complexes I and III and enhances ROS production: Results of a study on cell cultures. Mitochondrion 2010, 10, 487-496. [CrossRef] 
20. Pacelli, C.; De Rasmo, D.; Signorile, A.; Grattagliano, I.; di Tullio, G.; D’Orazio, A.; Nico, B.; Comi, G.P.; Ronchi, D.; Ferranini, E.; et al. Mitochondrial defect and PGC-1alpha dysfunction in parkin-associated familial Parkinson's disease. Biochim. Biophys. Acta 2011, 1812, 1041-1053. [CrossRef]

21. Zhang, J.; Zhang, Q. Using Seahorse Machine to Measure OCR and ECAR in Cancer Cells. Methods Mol. Biol. 2019, 1928, 353-363. [CrossRef]

22. Murphy, M.P. How mitochondria produce reactive oxygen species. Biochem. J. 2009, 417, 1-13. [CrossRef]

23. Chen, X.; Zhong, Z.; Xu, Z.; Chen, L.; Wang, Y. 2',7'-Dichlorodihydrofluorescein as a fluorescent probe for reactive oxygen species measurement: Forty years of application and controversy. Free Radic. Res. 2010, 44, 587-604. [CrossRef] [PubMed]

24. Schvartzman, J.M.; Thompson, C.B.; Finley, L.W.S. Metabolic regulation of chromatin modifications and gene expression. J. Cell Biol. 2018, 217, 2247-2259. [CrossRef] [PubMed]

25. Letts, J.A.; Sazanov, L.A. Clarifying the supercomplex: The higher-order organization of the mitochondrial electron transport chain. Nat. Struct Mol. Biol. 2017, 24, 800-808. [CrossRef] [PubMed]

26. Genova, M.L.; Lenaz, G. The Interplay Between Respiratory Supercomplexes and ROS in Aging. Antioxid. Redox Signal. 2015, 23, 208-238. [CrossRef] [PubMed]

27. Scarpulla, R.C. Transcriptional paradigms in mammalian mitochondrial biogenesis and function. Physiol. Rev. 2008, 88, 611-638. [CrossRef] [PubMed]

28. Handschin, C.; Spiegelman, B.M. Peroxisome proliferator-activated receptor gamma coactivator 1 coactivators, energy homeostasis, and metabolism. Endocr. Rev. 2006, 27, 728-735. [CrossRef]

29. Wenz, T. Regulation of mitochondrial biogenesis and PGC-1alpha under cellular stress. Mitochondrion 2013, 13, 134-142. [CrossRef]

30. Fan, W.; Evans, R. PPARs and ERRs: Molecular mediators of mitochondrial metabolism. Curr. Opin. Cell Biol. 2015, 33, 49-54. [CrossRef]

31. Suthammarak, W.; Yang, Y.Y.; Morgan, P.G.; Sedensky, M.M. Complex I function is defective in complex IV-deficient Caenorhabditis elegans. J. Biol. Chem. 2009, 284, 6425-6435. [CrossRef]

32. Protasoni, M.; Perez-Perez, R.; Lobo-Jarne, T.; Harbour, M.E.; Ding, S.; Penas, A.; Diaz, F.; Moraes, C.T.; Fearnley, I.M.; Zeviani, M.; et al. Respiratory supercomplexes act as a platform for complex III-mediated maturation of human mitochondrial complexes I and IV. EMBO J. 2020, 39, e102817. [CrossRef] [PubMed]

33. Lee, J.; Saha, P.K.; Yang, Q.H.; Lee, S.; Park, J.Y.; Suh, Y.; Lee, S.K.; Chan, L.; Roeder, R.G.; Lee, J.W. Targeted inactivation of MLL3 histone H3-Lys-4 methyltransferase activity in the mouse reveals vital roles for MLL3 in adipogenesis. Proc. Natl. Acad. Sci. USA 2008, 105, 19229-19234. [CrossRef] [PubMed]

34. Guo, C.; Chang, C.C.; Wortham, M.; Chen, L.H.; Kernagis, D.N.; Qin, X.; Cho, Y.W.; Chi, J.T.; Grant, G.A.; McLendon, R.E.; et al. Global identification of MLL2-targeted loci reveals MLL2's role in diverse signaling pathways. Proc. Natl. Acad. Sci. USA 2012, 109, 17603-17608. [CrossRef] [PubMed]

35. Park, J.H.; Zhuang, J.; Li, J.; Hwang, P.M. p53 as guardian of the mitochondrial genome. FEBS Lett. 2016, 590, 924-934. [CrossRef]

36. Park, J.Y.; Wang, P.Y.; Matsumoto, T.; Sung, H.J.; Ma, W.; Choi, J.W.; Anderson, S.A.; Leary, S.C.; Balaban, R.S.; Kang, J.G.; et al. p53 improves aerobic exercise capacity and augments skeletal muscle mitochondrial DNA content. Circ. Res. 2009, 105, 705-712. [CrossRef]

37. Matoba, S.; Kang, J.G.; Patino, W.D.; Wragg, A.; Boehm, M.; Gavrilova, O.; Hurley, P.J.; Bunz, F.; Hwang, P.M. p53 regulates mitochondrial respiration. Science 2006, 312, 1650-1653. [CrossRef]

38. Cela, O.; Scrima, R.; Pazienza, V.; Merla, G.; Benegiamo, G.; Augello, B.; Fugetto, S.; Menga, M.; Rubino, R.; Fuhr, L.; et al. Clock genes-dependent acetylation of complex I sets rhythmic activity of mitochondrial OxPhos. Biochim Biophys Acta 2016, 1863, 596-606. [CrossRef]

39. Scrima, R.; Cela, O.; Merla, G.; Augello, B.; Rubino, R.; Quarato, G.; Fugetto, S.; Menga, M.; Fuhr, L.; Relogio, A.; et al. Clock-genes and mitochondrial respiratory activity: Evidence of a reciprocal interplay. Biochim Biophys Acta 2016, 1857, 1344-1351. [CrossRef]

40. Koutsioumpa, M.; Hatziapostolou, M.; Polytarchou, C.; Tolosa, E.J.; Almada, L.L.; Mahurkar-Joshi, S.; Williams, J.; Tirado-Rodriguez, A.B.; Huerta-Yepez, S.; Karavias, D.; et al. Lysine methyltransferase 2D regulates pancreatic carcinogenesis through metabolic reprogramming. Gut 2019, 68, 1271-1286. [CrossRef]

41. Jiang, H.; Shukla, A.; Wang, X.; Chen, W.Y.; Bernstein, B.E.; Roeder, R.G. Role for Dpy-30 in ES cell-fate specification by regulation of H3K4 methylation within bivalent domains. Cell 2011, 144, 513-525. [CrossRef] 
42. Yang, Z.; Augustin, J.; Hu, J.; Jiang, H. Physical Interactions and Functional Coordination between the Core Subunits of Set1/Mll Complexes and the Reprogramming Factors. PLoS ONE 2015, 10, e0145336. [CrossRef]

43. Yang, Z.; Shah, K.; Khodadadi-Jamayran, A.; Jiang, H. Control of Hematopoietic Stem and Progenitor Cell Function through Epigenetic Regulation of Energy Metabolism and Genome Integrity. Stem Cell Reports 2019, 13, 61-75. [CrossRef] [PubMed]

44. Carosso, G.A.; Boukas, L.; Augustin, J.J.; Nguyen, H.N.; Winer, B.L.; Cannon, G.H.; Robertson, J.D.; Zhang, L.; Hansen, K.D.; Goff, L.A.; et al. Precocious neuronal differentiation and disrupted oxygen responses in Kabuki syndrome. JCI Insight 2019, 4.

45. Benjamin, J.S.; Pilarowski, G.O.; Carosso, G.A.; Zhang, L.; Huso, D.L.; Goff, L.A.; Vernon, H.J.; Hansen, K.D.; Bjornsson, H.T. A ketogenic diet rescues hippocampal memory defects in a mouse model of Kabuki syndrome. Proc. Natl. Acad. Sci. USA 2017, 114, 125-130. [CrossRef] [PubMed]

(C) 2020 by the authors. Licensee MDPI, Basel, Switzerland. This article is an open access article distributed under the terms and conditions of the Creative Commons Attribution (CC BY) license (http://creativecommons.org/licenses/by/4.0/). 